\title{
THE FILTRATION RATE, EFFECTIVE RENAL BLOOD FLOW, TUBULAR EXCRETORY MASS AND PHENOL RED CLEAR- ANCE IN SPECIFIC TOXEMIA OF PREGANANCY ${ }^{1}$
}

\author{
By IRWIN WELLEN, CATHERINE A. WELSH AND HOWARD C. TAYLOR, JR. \\ WITH THE TECHNICAL ASSISTANCE OF ANNA ROSENTHAL \\ (From the Department of Obstetrics and Gynecology, New York University College of Medicine \\ and the Obstetrical and Gynecological Service of the Third (New York University) \\ Surgical Division of Bellevue Hospital, New York City)
}

(Received for publication August 12, 1941)

The vascular and renal disorders of the last trimester of pregnancy are classified by the University Obstetrical Service in the following groups: specific toxemia, pre-existing essential hypertension, pre-existing glomerulonephritis and specific toxemia superimposed on pre-existing hypertension or nephritis (1).

Specific toxemia is clinically characterized by the rather abrupt appearance of hypertension, usually in the last trimester of a previously normal pregnancy. This rise in blood pressure is typically accompanied by edema and proteinuria. Hematuria and azotemia are not seen, but the convulsions of eclampsia may occur. The postpartum course of the disease likewise helps to identify it. Edema subsides soon after delivery. Proteinuria usually disappears in the puerperium, although traces of protein in the urine may be present for weeks. Blood pressure falls to a normal level in the majority soon after delivery, but it is a striking feature of such an attack that in many instances it is followed by permanent hypertension.

The present communication is an investigation of renal function in specific toxemia of pregnancy, based upon a study of inulin, phenol red and diodrast clearances and diodrast $T_{m}$. The inulin clearance is considered to be a measure of the rate of glomerular filtration, diodrast clearance a measure of effective renal blood flow and diodrast $T_{m}$ a measure of tubular excretory mass $(2,3)$, within the definitions and limitations accorded to these terms by Smith (4).

Using these methods, Goldring et al. $(5,6,7$, 8 ) have demonstrated that renal ischemia of varying degree, associated with an increase in filtration fraction which is indicative of efferent arteriolar

1 This study was made with the aid of a grant from the Commonwealth Fund. hypertonus, is usually present in essential hypertension in men and non-pregnant women. Chesley et al. (9) have shown that the effective renal blood flow in toxemia of pregnancy, as determined by the diodrast clearance of twenty patients, is the same as that of normal pregnant and nonpregnant women. Corcoran and Page (10), using clearances of inulin and phenol red, found the filtration rate diminished, effective renal blood flow normal and the phenol red/inulin clearance ratio high during toxemia in seven subjects when compared to their postpartum values. In three of these subjects, in whom diodrast clearance was determined both antepartum and postpartum, they observed a fall in diodrast clearance and a rise in filtration fraction after delivery. They attribute the antepartum decrease in filtration fraction to swelling of the glomerular basement membrane. That the glomerular filtration rate and effective renal blood flow are unaffected in normal pregnancy was concluded in our previous report (11). This was based on inulin and phenol red clearances in twenty normal pregnant women, with diodrast clearance in eleven and diodrast $T_{m}$ in eight.

\section{MATERIAL AND METHODS}

Thirteen patients, who had been seen early in pregnancy and found at that time to have no evidence of vascular or renal disease, have been studied in fourteen pregnancies complicated by specific toxemia. None gave a history of previous hypertension or kidney disease except for one patient who, though her blood pressure was normal between gestations, had developed a typical attack of specific toxemia in a previous pregnancy. In the last trimester, hypertension, proteinuria and edema appeared in all of these patients but, as may be seen in Tables I and II, proteinuria and edema had disappeared in some instances before the day of the antepartum tests. Two patients developed the typical antepartum convulsions of eclampsia and there were three intrauterine fetal deaths. 
These thirteen patients are divided into two groups depending upon the behavior of the blood pressure after delivery. One group is characterized by clinical cure, the other by residual hypertension. The classification of any patient was decided not at the time of the postpartum tests but in the follow-up toxemia clinic. The significance of this grouping with respect to renal function will be discussed.

\section{RESULTS}

The data on both groups appear in detail in Tables I and II. In eleven patients, inulin, phenol red and diodrast clearances, and diodrast $T_{m}$ were determined, while in three, phenol red and inulin clearances only were done. The tests were performed as previously outlined (11). Each clearance figure represents the average of at least three urine collection periods; each diodrast $T_{m}$ figure, an average of five periods. Urine flow was maintained at high levels by methods already described and any test in which the urine flow was below $2 \mathrm{cc}$. per minute is not reported.

The average antepartum and postpartum values for each individual appear in Figures 1, 2 and 3. The antepartum figure is shown by a solid circle and the postpartum by an open circle, the arrow connecting them indicating the direction of change taking place after delivery. The data on toxemic patients are displayed against the statistical background obtained from normal women. In compiling these control data, nine normal non-pregnant women observed by Goldring, Chasis, Ranges and Smith (12), and eight normal pregnant women observed by us (11), have been treated as a single group. The mean and standard deviation from the mean of inulin and diodrast clearances

TABLE I

Specific toxemia of pregnancy with clinical cure postpartum

\begin{tabular}{|c|c|c|c|c|c|c|c|c|c|c|c|c|c|c|c|c|c|c|}
\hline \multirow{2}{*}{ Sub- } & \multirow{2}{*}{$\begin{array}{l}\text { Anto- } \\
\text { par- } \\
\text { tum } \\
\text { or } \\
\text { post- } \\
\text { par- } \\
\text { tum }\end{array}$} & \multirow{2}{*}{$\begin{array}{l}\text { Bur- } \\
\text { face } \\
\text { area }\end{array}$} & \multirow{2}{*}{ Date } & \multirow{2}{*}{$\begin{array}{l}\text { Dura- } \\
\text { tion } \\
\text { of } \\
\text { preg- } \\
\text { nanoy } \\
\text { in } \\
\text { weeks } \\
\text { by } \\
\text { his- } \\
\text { tory }\end{array}$} & \multirow{2}{*}{$\begin{array}{l}\text { Time } \\
\text { in } \\
\text { weeks } \\
\text { before } \\
\text { or } \\
\text { after } \\
\text { de- } \\
\text { livery }\end{array}$} & \multirow{2}{*}{$\begin{array}{l}\text { Mean } \\
\text { blood } \\
\text { prew- } \\
\text { sure } \\
\text { during } \\
\text { test }\end{array}$} & \multirow{2}{*}{$\begin{array}{c}\text { Protein- } \\
\text { uris }\end{array}$} & \multicolumn{3}{|c|}{ Plasma clearances } & \multirow{2}{*}{$\begin{array}{l}\text { Effeo- } \\
\text { tive } \\
\text { rensal } \\
\text { blood } \\
\text { flow }\end{array}$} & \multirow{2}{*}{$\begin{array}{l}\text { Inulin/ } \\
\text { Phenol } \\
\text { red }\end{array}$} & \multirow{2}{*}{$\begin{array}{c}\begin{array}{c}\text { Filtra- } \\
\text { tion } \\
\text { fraction }\end{array} \\
\begin{array}{c}\text { Inulin/ } \\
\text { Diodrast }\end{array}\end{array}$} & \multirow{2}{*}{$\begin{array}{l}\text { Phenol } \\
\text { red/ } \\
\text { Diodrast }\end{array}$} & \multirow{2}{*}{$\underset{\text { drast }}{\text { Dio- }}$} & \multirow{2}{*}{$\begin{array}{l}\text { Effeo- } \\
\text { tive } \\
\text { renal } \\
\text { blood } \\
\text { flow/ } \\
T_{m D}\end{array}$} & \multirow{2}{*}{$\mid \begin{array}{l}C_{D} \\
T_{m_{D}}\end{array}$} & \multirow{2}{*}{$\begin{array}{l}C_{I N /} \\
T_{m_{D}}\end{array}$} \\
\hline & & & & & & & & 量 & $\frac{\overrightarrow{8}}{\overline{8}}$ & $\begin{array}{l}\text { 苟 } \\
\text { 帝 }\end{array}$ & & & & & & & & \\
\hline M.I. & $\begin{array}{l}\mathbf{A}-\mathbf{P} \\
\mathbf{P}-\mathbf{P}\end{array}$ & $\begin{array}{c}q . \\
m . \\
1.44\end{array}$ & $\begin{array}{l}\text { September 18, 1938(b) } \\
\text { September 26, 1938(b) } \\
\text { Ootober 6, 1938(b) }\end{array}$ & $\begin{array}{l}35 \\
37\end{array}$ & $\begin{array}{l}3 \\
1 \\
1\end{array}$ & $\begin{array}{l}176 / 106 \\
160 / 110 \\
160 / 110\end{array}$ & & $\begin{array}{r}c . p e \\
p e \\
\\
148 \\
86 \\
118\end{array}$ & $\begin{array}{l}517 \\
288 \\
369\end{array}$ & $m$ & $\begin{array}{l}\text { ce. per } \\
1.75 \\
\text { s. m. } \\
\text { per } \\
\text { minute }\end{array}$ & $\begin{array}{l}\text { per } \\
\text { cent }\end{array}$ & $\begin{array}{l}\text { per } \\
\text { cont }\end{array}$ & $\begin{array}{l}\text { per } \\
\text { cent }\end{array}$ & $\begin{array}{l}\operatorname{mgm.} \\
\text { iodine } \\
\text { per } \\
\text { minute }\end{array}$ & & & \\
\hline M.C. & $\begin{array}{l}\mathbf{A}-\mathbf{P} \\
\mathbf{P}-\mathbf{P}\end{array}$ & 1.88 & $\begin{array}{l}\text { Ootober 4, 1938(b) } \\
\text { Ootober 20, 1938(e) } \\
\text { December 13, 1938(a) }\end{array}$ & 38 & $\begin{array}{l}2 \\
1 \\
9\end{array}$ & $\begin{array}{l}168 / 96 \\
184 / 120 \\
128 / 90\end{array}$ & & $\begin{array}{l}85 \\
91 \\
94\end{array}$ & $\begin{array}{l}268 \\
813 \\
358\end{array}$ & & & $\begin{array}{l}31.7 \\
29.1 \\
26.6\end{array}$ & & & & & & \\
\hline M.A. & $\begin{array}{l}\mathbf{A}-\mathbf{P} \\
\mathbf{P}-\mathbf{P}\end{array}$ & 1.46 & $\begin{array}{l}\text { November 17, } 1938(\mathrm{~s}) \\
\text { March 2, } 1939 \text { (a) } \\
\text { July 25, 1939(c) }\end{array}$ & 30 & $\begin{array}{r}6 \\
9 \\
25\end{array}$ & $\begin{array}{l}140 / 110 \\
120 / 74 \\
116 / 70\end{array}$ & & $\begin{array}{r}85 \\
183 \\
102\end{array}$ & $\begin{array}{l}266 \\
308 \\
288\end{array}$ & $\begin{array}{l}458 \\
538 \\
556\end{array}$ & $\begin{array}{l}780 \\
846 \\
856\end{array}$ & $\begin{array}{l}32.0 \\
43.2 \\
35.4\end{array}$ & $\begin{array}{l}18.6 \\
24.7 \\
18.4\end{array}$ & $\begin{array}{l}58.1 \\
57.2 \\
51.8\end{array}$ & $\begin{array}{l}37.1 \\
35.1\end{array}$ & $\begin{array}{l}21.9^{*} \\
22.8 \\
24.4\end{array}$ & $\begin{array}{l}12.7^{*} \\
14.5 \\
15.0\end{array}$ & $\begin{array}{l}\mathbf{8 . 3 6} \\
\mathbf{3 . 5 9} \\
\mathbf{2 . 0 1}\end{array}$ \\
\hline M.8. & $\begin{array}{l}\mathbf{A}-\mathbf{P} \\
\mathbf{P}-\mathbf{P}\end{array}$ & 1.55 & $\begin{array}{l}\text { December 7, 1938(e) } \\
\text { Deoember 15, 1938(a) } \\
\text { December 21, 1938(a) } \\
\text { January 10, 1939(a) } \\
\text { January 16, 1939(b) } \\
\text { Maroh 6, 1939(d) }\end{array}$ & $\begin{array}{l}\mathbf{3 5} \\
\mathbf{3 6} \\
\mathbf{3 7}\end{array}$ & $\begin{array}{r}\mathbf{4} \\
\mathbf{3} \\
\mathbf{2} \\
\mathbf{2} \\
\mathbf{3} \\
\mathbf{1 0}\end{array}$ & $\begin{array}{l}154 / 110 \\
144 / 104 \\
180 / 120 \\
160 / 116 \\
158 / 116 \\
128 / 90\end{array}$ & & $\begin{array}{r}101 \\
88 \\
77 \\
118 \\
98 \\
106\end{array}$ & $\begin{array}{l}406 \\
403 \\
392 \\
409 \\
372 \\
301\end{array}$ & $\begin{array}{l}842 \\
792 \\
750 \\
658 \\
537 \\
618\end{array}$ & $\begin{array}{r}1367 \\
1207 \\
1133 \\
1024 \\
835 \\
977\end{array}$ & $\begin{array}{l}24.8 \\
20.4 \\
19.7 \\
28.9 \\
26.8 \\
35.8\end{array}$ & $\begin{array}{l}12.0 \\
10.4 \\
10.8 \\
17.9 \\
18.3 \\
17.2\end{array}$ & $\begin{array}{l}48.2 \\
50.9 \\
52.3 \\
62.2 \\
69.3 \\
48.7\end{array}$ & $\begin{array}{l}45.5 \\
41.0\end{array}$ & $\begin{array}{l}31.6^{*} \\
27.9^{*} \\
26.2^{*} \\
23.6^{*} \\
18.4^{*} \\
23.8^{\circ}\end{array}$ & $\begin{array}{l}19.5^{*} \\
18.3^{*} \\
17.8^{*} \\
15.2^{*} \\
11.8 \\
15.1\end{array}$ & $\begin{array}{l}2.33^{*} \\
1.89^{*} \\
1.78^{*} \\
2.72^{*} \\
2.15 \\
\mathbf{2 . 8 9}\end{array}$ \\
\hline \multirow[t]{2}{*}{ E.8. } & A-P & 1.7 & $\begin{array}{l}\text { March 15, } 1939(\mathrm{a}) \\
\text { April 6, } 1989(\mathrm{o}) \\
\text { July 6, } 1939(\mathrm{~d})\end{array}$ & 37 & $\begin{array}{r}2 \\
2 \\
11\end{array}$ & $\begin{array}{l}136 / 108 \\
122 / 86 \\
130 / 88\end{array}$ & $\underset{0}{0}$ & $\begin{array}{l}155 \\
128 \\
157\end{array}$ & $\begin{array}{l}523 \\
388 \\
433\end{array}$ & $\begin{array}{l}776 \\
517 \\
658\end{array}$ & $\begin{array}{r}1257 \\
872 \\
1042\end{array}$ & $\begin{array}{l}29.6 \\
31.7 \\
36.3\end{array}$ & $\begin{array}{l}20.0 \\
23.8 \\
24.1\end{array}$ & $\begin{array}{l}67.4 \\
75.1 \\
66.4\end{array}$ & $\begin{array}{l}68.2 \\
67.3 \\
62.0\end{array}$ & $\begin{array}{l}18.4 \\
18.0 \\
16.8\end{array}$ & $\begin{array}{c}11.4 \\
7.68 \\
10.5\end{array}$ & $\begin{array}{l}2.27 \\
1.83 \\
2.58\end{array}$ \\
\hline & $\begin{array}{l}\mathbf{A}-\mathbf{P} \\
\mathbf{P}-\mathbf{P}\end{array}$ & 1.7 & $\begin{array}{l}\text { Novermber 25, } 1940 \text { (c) } \\
\text { December 23, } 1940 \text { (o) }\end{array}$ & 35 & $\begin{array}{l}3 \\
1\end{array}$ & $\begin{array}{l}140 / 100 \\
118 / 90\end{array}$ & $\begin{array}{l}\mathbf{0} \\
\mathbf{0}\end{array}$ & $\begin{array}{l}158 \\
176\end{array}$ & $\begin{array}{l}476 \\
534\end{array}$ & $\begin{array}{l}765 \\
788\end{array}$ & $\begin{array}{l}1280 \\
1175\end{array}$ & $\begin{array}{l}32.2 \\
38.0\end{array}$ & $\begin{array}{l}20.0 \\
23.9\end{array}$ & $\begin{array}{l}62.8 \\
72.4\end{array}$ & $\begin{array}{l}61.0 \\
70.0\end{array}$ & $\begin{array}{l}21.0 \\
16.8\end{array}$ & $\begin{array}{l}12.4 \\
10.5\end{array}$ & $\begin{array}{l}\mathbf{8 . 4 9} \\
\mathbf{2 . 5 8}\end{array}$ \\
\hline F.P. & $\begin{array}{l}\mathbf{A}-\mathbf{P} \\
\mathbf{P}-\mathbf{P}\end{array}$ & 1.65 & $\begin{array}{l}\text { March 28, } 1939(\mathrm{c}) \\
\text { April 3, } 1939(\mathrm{o}) \\
\text { April 18, } 1939(\mathrm{o}) \\
\text { June 8, } 1939 \text { (d) }\end{array}$ & $\begin{array}{l}31 \\
32 \\
34\end{array}$ & $\begin{array}{l}9 \\
8 \\
6 \\
2\end{array}$ & $\begin{array}{l}148 / 100 \\
160 / 110 \\
160 / 114 \\
140 / 86\end{array}$ & & $\begin{array}{r}80 \\
84 \\
101 \\
117\end{array}$ & $\begin{array}{l}\mathbf{8 2 8} \\
\mathbf{3 7 9} \\
487 \\
\mathbf{3 7 7}\end{array}$ & $\begin{array}{l}589 \\
686 \\
651 \\
540\end{array}$ & $\begin{array}{r}924 \\
1048 \\
1100 \\
787\end{array}$ & $\begin{array}{l}24.8 \\
22.8 \\
28.1 \\
31.0\end{array}$ & $\begin{array}{l}13.7 \\
13.2 \\
15.5 \\
21.7\end{array}$ & $\begin{array}{l}55.5 \\
59.6 \\
67.1 \\
69.8\end{array}$ & $\begin{array}{l}44.1 \\
45.4 \\
46.0\end{array}$ & $\begin{array}{l}21.0 \\
28.0 \\
24.5 \dagger \\
17.1\end{array}$ & $\begin{array}{l}13.8 \\
14.0 \\
14.0 \dagger \\
11.7\end{array}$ & $\begin{array}{l}1.81 \\
1.85 \\
2.25 t \\
2.84\end{array}$ \\
\hline F.8. & A-P & 1.78 & $\begin{array}{l}\text { Ootober } 25,1839(0) \\
\text { November } 13,1839(\mathrm{c})\end{array}$ & 39 & $\frac{2}{2}$ & $\begin{array}{l}138 / 98 \\
124 / 84\end{array}$ & $+t+$ & $\begin{array}{r}100 \\
92\end{array}$ & $\begin{array}{l}407 \\
337\end{array}$ & $\begin{array}{l}668 \\
468\end{array}$ & $\begin{array}{l}940 \\
770\end{array}$ & $\begin{array}{l}24.6 \\
27.3\end{array}$ & $\begin{array}{l}15.0 \\
19.7\end{array}$ & $\begin{array}{l}60.9 \\
72.1\end{array}$ & $\begin{array}{l}40.7 \\
55.1\end{array}$ & $\begin{array}{l}18.9 \\
14.0\end{array}$ & 18.4 & $\begin{array}{l}2.01 \\
1.67\end{array}$ \\
\hline
\end{tabular}

* Postpartum $T_{m}$.

† Antepartum $T_{m}$.

(a) Infusion with physiological saline.

(b) Infusion with physiological saline and sodium sulphate.

(c) Infusion with mannitol in distilled water.

(d) Infusion with sorbitol in distilled water.

(e) Infusion with $\mathbf{5}$ per cent glucose in distilled water. 
TABLE II

Specific toxemia of pregnancy with residual hypertension postpartum

\begin{tabular}{|c|c|c|c|c|c|c|c|c|c|c|c|c|c|c|c|c|c|c|}
\hline \multirow{2}{*}{$\begin{array}{c}\text { Sub- } \\
\text { ject }\end{array}$} & \multirow{2}{*}{$\begin{array}{l}\text { Anto- } \\
\text { par- } \\
\text { tum } \\
\text { or } \\
\text { post- } \\
\text { par- } \\
\text { tum }\end{array}$} & \multirow{2}{*}{$\left|\begin{array}{l}\text { Sur- } \\
\text { faco } \\
\text { area }\end{array}\right|$} & \multirow{2}{*}{ Date } & \multirow{2}{*}{$\begin{array}{c}\text { Dura- } \\
\text { tion } \\
\text { of } \\
\text { preg- } \\
\text { nanoy } \\
\text { in } \\
\text { weeks } \\
\text { by } \\
\text { his- } \\
\text { tory }\end{array}$} & \multirow{2}{*}{$\begin{array}{c}\text { Time } \\
\text { in } \\
\text { weeks } \\
\text { before } \\
\text { or } \\
\text { after } \\
\text { do- } \\
\text { livery }\end{array}$} & \multirow{2}{*}{$\begin{array}{l}\text { Mean } \\
\text { blood } \\
\text { pres } \\
\text { sure } \\
\text { during } \\
\text { test }\end{array}$} & \multirow{2}{*}{$\begin{array}{c}\text { Protein- } \\
\text { uria }\end{array}$} & \multicolumn{3}{|c|}{ Plasma clearances } & \multirow{2}{*}{$\begin{array}{l}\text { Effec- } \\
\text { tive } \\
\text { renal } \\
\text { blood } \\
\text { flow }\end{array}$} & \multirow{2}{*}{$\begin{array}{c}\text { Inulin/ } \\
\text { Phenol } \\
\text { red }\end{array}$} & \multirow{2}{*}{$\begin{array}{c}\begin{array}{c}\text { Filtra- } \\
\text { tion } \\
\text { fraction }\end{array} \\
\begin{array}{c}\text { Inulin/ } \\
\text { Diodrast }\end{array}\end{array}$} & \multirow{2}{*}{$\begin{array}{l}\text { Phenol } \\
\text { red/ } \\
\text { Diodrast }\end{array}$} & \multirow{2}{*}{$\begin{array}{c}\text { Dio- } \\
\text { drast } \\
T_{m}\end{array}$} & \multirow{2}{*}{\begin{tabular}{|c} 
Effec- \\
tive \\
renal \\
blood \\
flow/ \\
$T_{m_{D}}$
\end{tabular}} & \multirow{2}{*}{$\left|\begin{array}{l}C_{D I} \\
T_{m D}\end{array}\right|$} & \multirow{2}{*}{$\begin{array}{l}C_{I N} \\
T_{m_{D}}\end{array}$} \\
\hline & & & & & & & & 豆 & 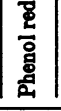 & 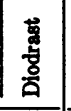 & & & & & & & & \\
\hline G.C. & A-P & m. & $\begin{array}{l}\text { November 15, 1938(a) } \\
\text { November 28, 1938(a) }\end{array}$ & 39 & $\frac{1}{2}$ & $\begin{array}{l}132 / 100 \\
134 / 96 \\
\end{array}$ & $\begin{array}{l}\mathbf{0} \\
\mathbf{0}\end{array}$ & $\begin{array}{r}62 \\
118 \\
\end{array}$ & \begin{tabular}{|l|}
228 \\
290 \\
\end{tabular} & sq. $m$. & $\begin{array}{l}\text { c.per } \\
1.7 s \\
\text { oq. } m . \\
\text { per } \\
\text { minute }\end{array}$ & $\begin{array}{l}\text { per } \\
\text { cent } \\
\\
27.2 \\
40.7 \\
\end{array}$ & $\begin{array}{l}\text { per } \\
\text { cent }\end{array}$ & $\begin{array}{l}\text { per } \\
\text { cent }\end{array}$ & $\begin{array}{c}\operatorname{mom} \\
\text { iodine } \\
\text { per } \\
\text { minute }\end{array} \mid$ & & & \\
\hline M.C. & $\begin{array}{l}\mathbf{A}-\mathbf{P} \\
\mathbf{P}-\mathbf{P}\end{array}$ & 1.8 & \begin{tabular}{|l|} 
January 19, 1939(e) \\
January 24, 1939(e) \\
February 10, 1939(d) \\
February 21, 1939(d)
\end{tabular} & $\begin{array}{l}36 \\
37 \\
39\end{array}$ & $\begin{array}{l}4 \\
3 \\
1 \\
2\end{array}$ & \begin{tabular}{|l|}
$160 / 122$ \\
$150 / 124$ \\
$150 / 20$ \\
$134 / 98$ \\
\end{tabular} & $\begin{array}{c}++ \\
++ \\
++\end{array}$ & $\begin{array}{l}50 \\
41 \\
44 \\
96\end{array}$ & \begin{tabular}{l|}
183 \\
153 \\
196 \\
259 \\
\end{tabular} & $\begin{array}{l}228 \\
207 \\
288 \\
333 \\
\end{array}$ & $\begin{array}{l}374 \\
342 \\
469 \\
503 \\
\end{array}$ & $\begin{array}{l}27.3 \\
26.8 \\
22.4 \\
37.1 \\
\end{array}$ & $\begin{array}{l}21.9 \\
19.8 \\
15.3 \\
28.8\end{array}$ & $\begin{array}{l}80.2 \\
73.9 \\
68.1 \\
77.8\end{array}$ & $\begin{array}{l}31.5 \\
48.8\end{array}$ & $\begin{array}{l}11.9^{*} \\
10.9^{*} \\
14.9 \\
10.3 \\
\end{array}$ & $\begin{array}{l}7.24^{*} \\
6.57^{*} \\
9.14 \\
6.82 \\
\end{array}$ & $\begin{array}{l}1.59^{*} \\
1.3^{*} \\
1.4 \\
1.97\end{array}$ \\
\hline H.F. & $\begin{array}{l}\text { A-P } \\
\mathbf{P}-\mathbf{P}\end{array}$ & 1.44 & $\begin{array}{l}\text { January 26, 1939(e) } \\
\text { February 2,1939(a) } \\
\text { February 14, 1939(d) } \\
\text { February 27, 1939(d) } \\
\text { November 29, 1939(a) }\end{array}$ & $\begin{array}{l}31 \\
32 \\
34\end{array}$ & $\begin{array}{r}4 \\
3 \\
1 \\
2 \\
41\end{array}$ & \begin{tabular}{|l|}
$160 / 120$ \\
$158 / 112$ \\
$190 / 136$ \\
$192 / 134$ \\
$150 / 106$ \\
\end{tabular} & $\begin{array}{c}0 \\
+ \\
++ \\
0 \\
0\end{array}$ & $\begin{array}{l}143 \\
152 \\
104 \\
121 \\
125 \\
\end{array}$ & $\begin{array}{l}440 \\
409 \\
412 \\
319 \\
329 \\
\end{array}$ & $\begin{array}{l}825 \\
785 \\
752 \\
494 \\
596\end{array}$ & $\begin{array}{r}1290 \\
1235 \\
1360 \\
856 \\
983\end{array}$ & $\begin{array}{l}32.5 \\
37.2 \\
25.2 \\
37.9 \\
38.0\end{array}$ & $\begin{array}{l}17.3 \\
19.4 \\
13.8 \\
24.5 \\
21.0\end{array}$ & $\begin{array}{l}53.4 \\
52.1 \\
54.8 \\
64.6 \\
55.2\end{array}$ & $\begin{array}{l}36.7 \\
48.4 \\
42.6 \\
36.3\end{array}$ & \begin{tabular}{|l|}
$30.3^{*}$ \\
33.7 \\
28.1 \\
20.1 \\
27.1
\end{tabular} & $\begin{array}{l}19.4^{*} \\
21.4 \\
15.5 \\
11.6 \\
16.4\end{array}$ & \begin{tabular}{|l}
3.36 \\
4.14 \\
2.15 \\
2.84 \\
3.44
\end{tabular} \\
\hline M.S. & $\begin{array}{l}\text { A-P } \\
\text { P-P }\end{array}$ & 1.24 & $\begin{array}{l}\text { March 23, 1939(d) } \\
\text { March 30, 1939(c) } \\
\text { May 15, 1939(c) }\end{array}$ & $\begin{array}{l}35 \\
36\end{array}$ & $\begin{array}{l}2 \\
1 \\
6\end{array}$ & \begin{tabular}{l|}
$160 / 110$ \\
$162 / 110$ \\
$130 / 90$ \\
\end{tabular} & $\begin{array}{c}++ \\
+\end{array}$ & $\begin{array}{l}89 \\
85 \\
92 \\
\end{array}$ & \begin{tabular}{l|}
279 \\
278 \\
237 \\
\end{tabular} & $\begin{array}{l}535 \\
481 \\
384\end{array}$ & $\begin{array}{l}854 \\
690 \\
652\end{array}$ & $\begin{array}{l}31.9 \\
30.6 \\
38.8\end{array}$ & $\begin{array}{l}16.6 \\
17.7 \\
24.0\end{array}$ & $\begin{array}{l}52.2 \\
57.8 \\
61.7\end{array}$ & $\begin{array}{l}36.5 \\
35.8\end{array}$ & $\begin{array}{l}23.4 \\
19.3 \\
18.0^{*} \\
\end{array}$ & \begin{tabular}{l|}
14.7 \\
13.4 \\
$10.6^{*}$
\end{tabular} & $\begin{array}{l}2.44 \\
2.37 \\
2.54\end{array}$ \\
\hline H.S. & $\begin{array}{l}\text { A-P } \\
\text { P-P }\end{array}$ & 1.97 & $\begin{array}{l}\text { May 23, 1939(c) } \\
\text { June 10, } 1939 \text { (d) } \\
\text { June 29, } 1939 \text { (d) }\end{array}$ & $\begin{array}{l}35 \\
37\end{array}$ & $\begin{array}{l}4 \\
2 \\
2 \\
\end{array}$ & $\begin{array}{l}144 / 104 \\
130 / 90 \\
130 / 98 \\
\end{array}$ & $\underset{+t+}{+t}$ & $\begin{array}{l}106 \\
122 \\
119 \\
\end{array}$ & \begin{tabular}{l|}
331 \\
376 \\
299 \\
\end{tabular} & $\begin{array}{l}583 \\
574 \\
\mathbf{4 4 8}\end{array}$ & $\begin{array}{l}908 \\
855 \\
666\end{array}$ & $\begin{array}{l}32.0 \\
32.5 \\
39.8\end{array}$ & $\begin{array}{l}18.2 \\
21.3 \\
26.6\end{array}$ & $\begin{array}{l}56.8 \\
65.5 \\
66.7\end{array}$ & $\begin{array}{l}42.4 \\
40.0\end{array}$ & \begin{tabular}{|l|}
21.4 \\
21.4 \\
$16.2^{*}$
\end{tabular} & $\mid \begin{array}{l}13.8 \\
14.3 \\
10.8^{*}\end{array}$ & $\begin{array}{l}2.5 \\
3.05 \\
2.89\end{array}$ \\
\hline A.F. & A-P & 1.56 & $\begin{array}{l}\text { June 1, 1939(c) } \\
\text { June 14, 1939(d) }\end{array}$ & 42 & $\begin{array}{l}1 \\
2\end{array}$ & $\begin{array}{l}134 / 90 \\
162 / 94\end{array}$ & + & $\begin{array}{l}105 \\
115\end{array}$ & $\begin{array}{l}391 \\
318\end{array}$ & $\begin{array}{l}743 \\
431\end{array}$ & $\begin{array}{r}1135 \\
645\end{array}$ & $\begin{array}{l}26.9 \\
36.2\end{array}$ & $\begin{array}{l}14.1 \\
26.7\end{array}$ & $\begin{array}{l}52.6 \\
73.8\end{array}$ & $\begin{array}{l}54.2 \\
46.4\end{array}$ & \begin{tabular}{|l|}
20.9 \\
13.9
\end{tabular} & $\begin{array}{l}13.7 \\
9.29\end{array}$ & $\begin{array}{l}1.94 \\
2.48\end{array}$ \\
\hline
\end{tabular}

* Antepartum $T_{m}$.

(a) Infusion with physiological saline.

(b) Infusion with physiological saline and sodium sulphate. $\quad$ (d) Infusion with sorbitol in distilled water

(c) Infusion with mannitol in distilled water.

(e) Infusion with 5 per cent glucose in distilled water.

and diodrast $T_{m}$ for the series of seventeen normal women have been calculated, using each individual once.

Diodrast $\mathrm{T}_{\mathrm{m}}$. Tubular excretory function in toxemia is typically unaffected, for the values for diodrast $T_{m}$ are distributed about equally above and below the normal mean. In only one instance (M. C., Table II) does the diodrast $T_{m}$ fall below the lowest normal value, and in this patient it returns to normal after delivery.

Inulin clearance. The inulin clearance per unit of diodrast $T_{m}$ appears in Figure 1, the heavy line representing the mean normal value, the light lines once and twice the standard deviation. The antepartum and postpartum observations are within normal limits with one exception. ${ }^{2}$ There is, however, an increase in filtration rate after

2 Inulin, phenol red and diodrast clearance and diodrast $T_{m}$ in M. C. (Table II), and inulin and phenol red clearance in G. C. (Table II), all of which were well below normal antepartum, rose considerably after delivery. Corcoran and Page report a similar case (10). delivery in all but three of these individuals, suggesting that during toxemia the filtration rate is reduced.

Diodrast clearance. The diodrast clearance per unit of diodrast $T_{m}$ (Figure 2) is above the mean normal line antepartum in eight of eleven subjects. The antepartum observations, with the exception of M. C. mentioned above, show the effective renal blood flow in toxemia to be normal; in some instances it is distinctly above normal. The two points above the $+2 \sigma$ line are the highest values of diodrast clearance, per unit of diodrast $T_{m}$, found in any of the women examined by Goldring et al. or by ourselves $(11,12)$.

In nine of eleven patients the diodrast clearance falls after delivery. This occurs in some cases even before the fall of blood pressure. This drop in diodrast clearance after delivery is evident both in the cured group and in the group with residual hypertension, but is greater in the latter.

Filtration fraction. In Figure 3 the filtration fraction is shown in relation to the diodrast clear- 


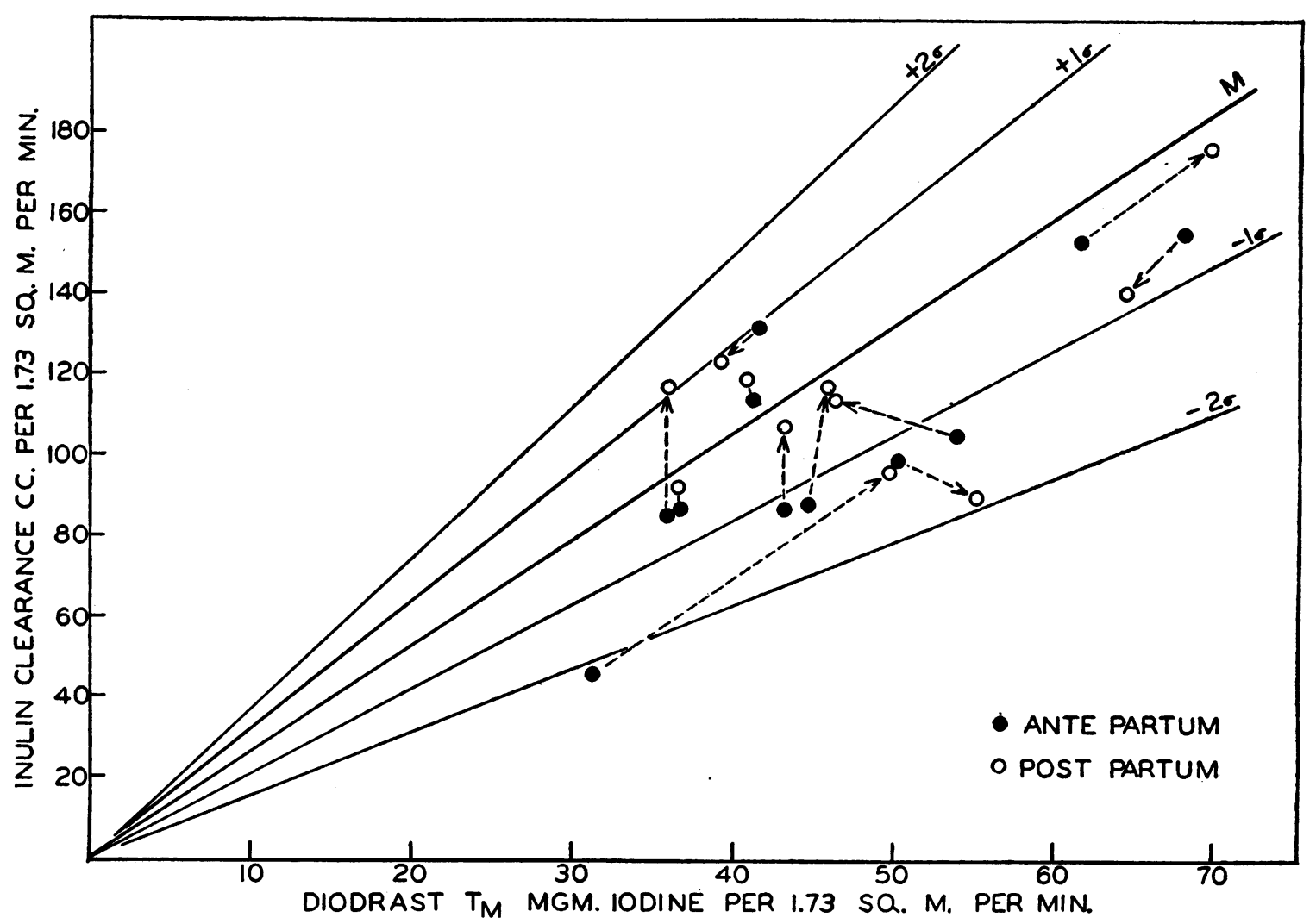

Fig. 1. Comparison of Antepartum With Postpartum Inulin Clearances

ance per unit of diodrast $T_{m}$. The horizontal line at 0.20 represents the mean normal value for the filtration fraction. The vertical line at $\mathbf{1 3 . 2}$ is the mean normal value of diodrast clearance per unit of diodrast $T_{m}$. The central curved line is the course of the variables if the glomerular filtration rate maintains its mean normal value at varying rates of renal plasma flow. The parallel curved lines are $+2 \sigma$ and $-2 \sigma$.

The figures for filtration fraction before delivery are below the lowest normal value in only five of eleven subjects. Yet it can be seen that all of the antepartum filtration fractions are on or below the normal line and that all of the arrows point upwards, showing the increase in filtration fraction after delivery. The antepartum reduction in filtration fraction is dependent upon an increase in diodrast clearance, a reduction in inulin clearance, or both. After delivery it rises without exception, in part because of an increased inulin clearance and in part because of a decreased diodrast clearance, the postpartum rise being greater in the group with persistent hypertension.
Phenol red clearance. The clearance of phenol red (Tables I and II), like that of diodrast, suffers a reduction after delivery, but to a lesser extent. It can be seen also that the inulin/phenol red clearance ratio is somewhat reduced antepartum and increased after delivery, a phenomenon not observed in normal pregnancy (11). Although the variations from the normal in this ratio are small, they follow the changes in filtration fraction in direction and time and may be considered to have the same functional significance.

Although the phenol red/diodrast clearance ratio varies over a wide range in antepartum and postpartum observations, it rises consistently after delivery in all instances where the diodrast clearance falls. This increase in ratio accompanying a decrease in diodrast clearance is in agreement with the observation of Chasis et al. that the phenol red/diodrast clearance ratio varies inversely as the renal blood flow (13). Calculation of renal blood flow from phenol red clearance in subjects with toxemia of pregnancy, especially 


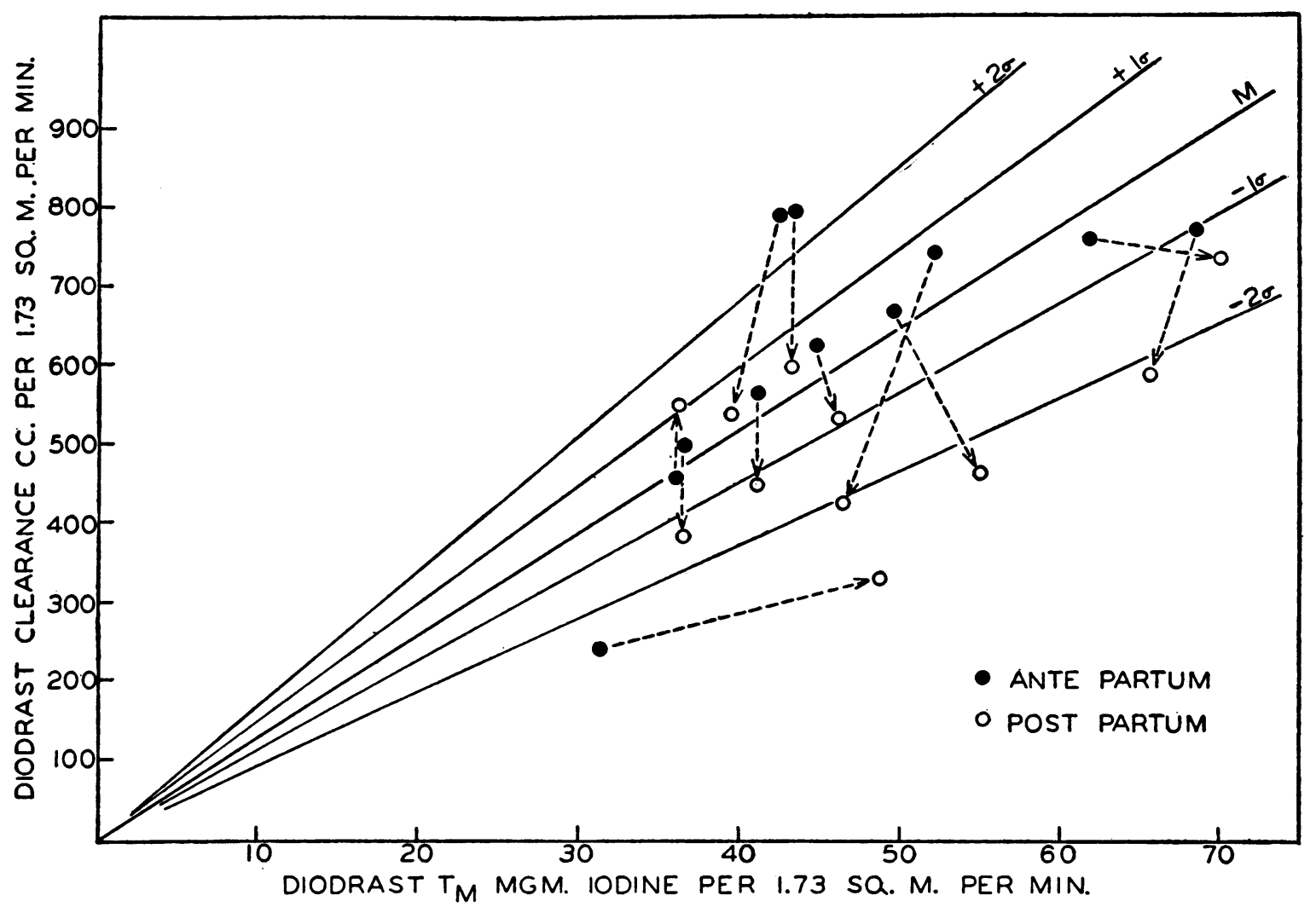

Fig. 2. Comparison of Antepartum With Postpartum Diodrast Clearances

for a comparison of antepartum with postpartum renal function, is evidently subject to greater error than is such calculation in normal subjects.

\section{DISCUSSION}

When renal function in fourteen pregnancies complicated by specific toxemia is compared to that of the control group, the clearances are found below the normal range in only two, while the filtration fraction is below normal in five. In other words, if judged from antepartum observations alone, one-half of this series of toxemic patients would appear to have normal kidney function. However, when these patients are considered as a group in comparison with the normals, as well as when antepartum figures are compared with postpartum figures, trends become evident which are not found in the pregnancy or puerperium of normal women. Before delivery there is a slight reduction in filtration rate and filtration fraction associated with a normal or slightly elevated effective renal blood flow. Fol- lowing delivery the filtration fraction rises in every instance, in part because of a rise in filtration rate and in part because of a fall in effective renal blood flow. In the group with residual hypertension the increase in filtration fraction is more marked than in the group with clinical cure and reaches levels seen in essential hypertension $(5,6,8)$.

The antepartum clearances and clearance ratios bear no consistent relation to the severity of the clinical manifestations or to the eventual outcome of the disease. The postpartum observations, however, fall readily into two groups and may offer some aid in prognosis. In the patients in whom hypertension was to persist, the diodrast clearance had fallen and the filtration fraction had risen considerably in the first test done postpartum, whereas in the cured group the inulin clearance had increased, the diodrast clearance had fallen slightly, and the filtration fraction had risen to normal in the first test done after delivery, regardless of whether the blood pressure had fallen to normal or not. 


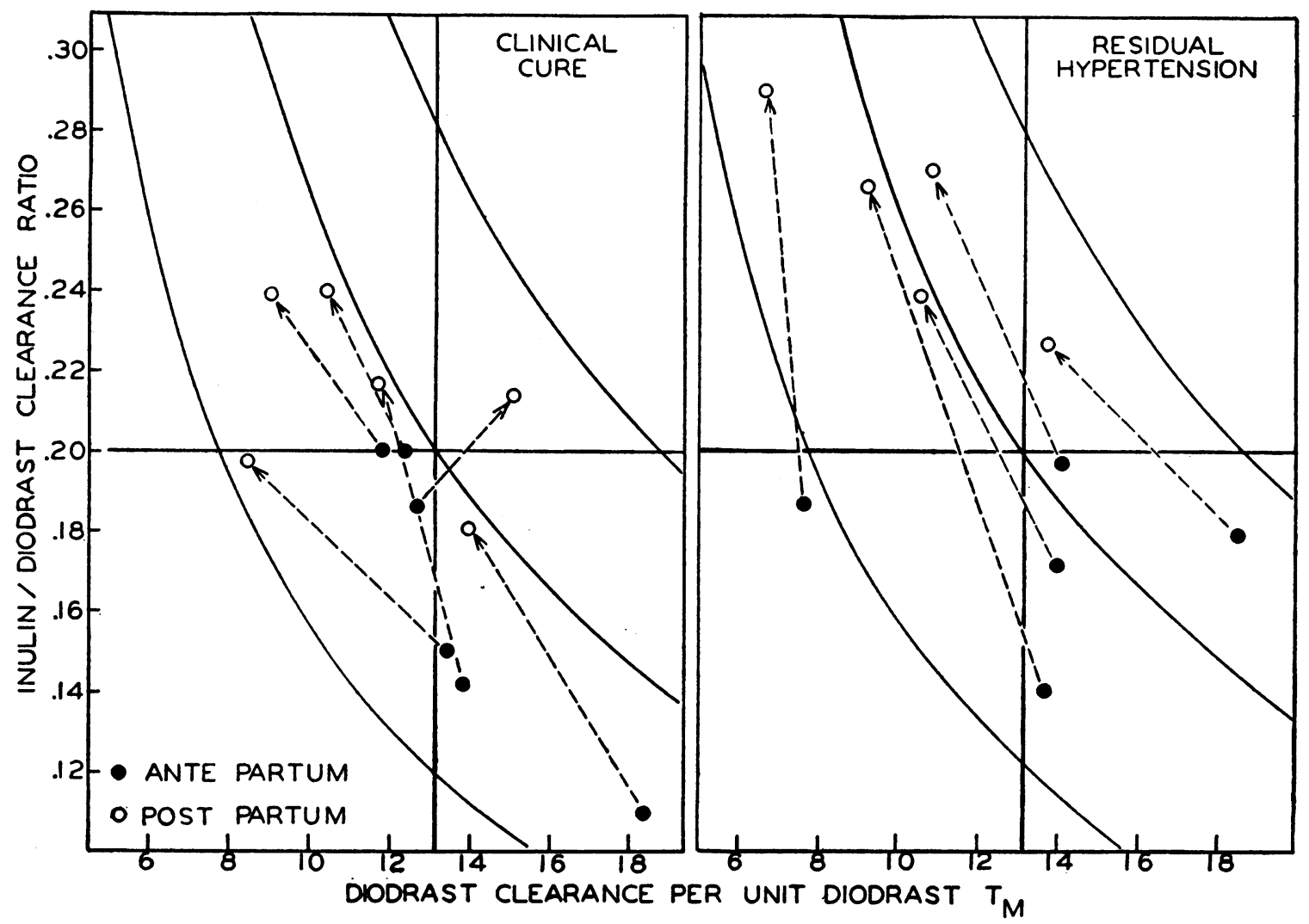

Fig. 3. Comparison of Antepartum With Postpartum Filtration Fractions

The slight diminution in filtration rate antepartum could result from afferent arteriolar constriction, which would cause a fall in filtration pressure, from edema of the kidney with resultant increase in interstitial pressure or from thickening of the basement membrane of the glomerulus with an increase in resistance to filtration (14). Any one of these mechanisms could conceivably be operative antepartum and disappear within a few days after delivery.

That the inulin clearance remains a true measure of glomerular filtration rate in toxemia is open to possible question on the ground that the permeability of the supposedly thickened glomerular filtering bed to the large inulin molecule might be reduced. In five subjects, however, on whom clearances of sorbitol or mannitol were done (15), the clearances of these hexitols were found to be identical with the simultaneous clearance of inulin. ${ }^{8}$ If inulin is not filtered at the same rate as

8 Hexitol and inulin clearances both antepartum and postpartum on patients F. P. (Table I), and H. F. and these smaller molecules, the hexitol/inulin clearance ratio can otherwise be maintained at the observed value of 1.0 only through the unlikely circumstance that a fraction of the filtered hexitol, exactly equal to that of the unfiltered inulin, is reabsorbed by the tubules. Yet tubular function in these five subjects is within normal limits as measured by diodrast $T_{m}$.

The observed alterations in effective renal blood flow are apparently not solely dependent upon the arterial blood pressure, for in some instances the diodrast clearance falls before the blood pressure falls. This observation indicates that the relatively high effective renal blood flow before delivery, and its decrease promptly thereafter, must be related to alterations in the renal vessels. The changes in effective renal blood flow may be explained by variations in efferent arteriolar tone (14), namely, dilatation of slight degree ante-

M. S. (Table II), and postpartum on E. S. (Table I) and M. C. (Table II), have already been published by Smith, Finkelstein and Smith (15). 
partum followed after delivery by a return to normal tone in the cured group and by spasm in the hypertensive group.

The dramatic improvement in the toxemic patient after delivery or fetal death is well known. The changes observed in diodrast clearances and filtration fraction-namely, a reduction from the level of normal renal blood flow or actual renal hyperemia to a level of renal ischemia accompanied by evidence of efferent arteriolar spasmare seen soon after delivery. Both of these phenomena are evidently associated with the emptying of the uterus or the cessation of placental circulation. In those pregnant patients in whom hypertension is to persist and in whom the efferent hypertonus characteristic of essential hypertension is to appear after delivery, it is possible that constriction of the efferent arteriole is prevented by some unknown factor effective so long as pregnancy continues. Such an hypothesis is supported by the fact that in a small series of women with essential hypertension (unpublished data) renal blood flow is higher antepartum than postpartum and in two individuals is higher during pregnancy than before pregnancy. The filtration rate in these women with essential hypertension is unaffected by pregnancy.

In respect to the genesis of hypertension in toxemia of pregnancy, both in patients whose blood pressure is to return to normal and in those in whom hypertension is to persist, there is no evidence that renal ischemia plays any part. Indeed, when the blood pressure is highest, there is neither the renal ischemia nor the efferent arteriolar hypertonus characteristic of essential hypertension. Yet those patients in whom hypertension persists show in their postpartum clearances renal disturbances which cannot be distinguished from those of essential hypertension. The failure to find a reduction in renal blood flow in toxemia of pregnancy in which hypertension is such a striking feature is an argument against the theory that renal ischemia is a primary causal factor of the hypertensive process.

\section{CONCLUSIONS}

1. In toxemia of pregnancy the tubular excretory mass (diodrast $T_{m}$ ) is normal; the effective renal blood flow (diodrast clearance) is normal or above normal; the glomerular filtration rate (inulin clearance) is somewhat reduced when referred to postpartum values; the filtration fraction is normal or low.

2. Following delivery the filtration fraction increases, in part because of a fall in diodrast clearance and in part because of an increase in inulin clearance. In the group with clinical cure these changes leave the figures within the normal range. In the group with persistent hypertension the results of these functional tests are identical with those found in essential hypertension.

3. The view that renal ischemia is an essential factor in the production of hypertension is opposed by the evidence that in the presence of the hypertension of toxemia there is a normal or even an increased renal blood flow.

We are indebted to Dr. Homer W. Smith for having many analyses of diodrast iodine carried out in the $\mathrm{De}$ partment of Physiology before the method was set up in this Laboratory. We wish further particularly to express our thanks to him for his interest and advice.

\section{BIBLIOGRAPHY}

1. Goldring, W., Lectures on Nephritis and Hypertension. Edwards Brothers, Inc., Ann Arbor, Mich., 1937.

2. Smith, H. W., The Physiology of the Kidney. Oxford University Press, New York, 1937.

3. Smith, H. W., Goldring, W., and Chasis, H., The measurement of the tubular excretory mass, effective blood flow and filtration rate in the normal human kidney. J. Clin. Invest., 1938, 17, 263.

4. Smith, H. W., Notes on the interpretation of clearance methods in the diseased kidney. J. Clin. Invest., 1941, 20, 631.

5. Goldring, W., Chasis, H., Ranges, H. A., and Smith, H. W., Effective renal blood flow and functional excretory mass in essential hypertension. J. Clin. Invest., 1938, 17, 505.

6. Goldring, W., Chasis, H., Ranges, H. A., and Smith, H. W., Effective renal blood flow in subjects with essential hypertension. J. Clin. Invest., 1941, 20 , 637.

7. Smith, H. W., Physiology of the Kidney. Porter Lectures. Series IX, Univ. Extension Div., Univ. of Kan., Lawrence, 1939.

8. Smith, H. W., Goldring, W., Chasis, H., and Ranges, H. A., Observations on the effective renal blood flow and functional excretory mass in man with special reference to essential hypertension. Am. J. Physiol., 1938, 123, 189.

9. Chesley, L. C., Connell, E. J., Chesley, E. R., Katz, J. D., and Glisson, C. S., The diodrast clearance 
and renal blood flow in toxemias of pregnancy. J. Clin. Invest., 1940, 19, 219.

10. Corcoran, A. C., and Page, I. H., Renal function in the late toxemias of pregnancy. Am. J. M. Sc., 1941, 201, 385.

11. Welsh, C. A., Wellen, I., and Taylor, H. C., Jr., The filtration rate, effective renal blood flow, tubular excretory mass and phenol red clearance in normal pregnancy. J. Clin. Invest., 1942, 21, 57.

12. Goldring, W., Chasis, H., Ranges, H. A., and Smith, H. W., Relations of effective renal blood flow and glomerular filtration to tubular excretory mass in normal men. J. Clin. Invest., 1940, 19, 739.
13. Chasis, H., Ranges, H. A., Goldring, W., and Smith, H. W., The control of renal blood flow and glomerular filtration in normal man. J. Clin. Invest., 1938, 17, 683.

14. Smith, H. W., Chasis, H., Goldring, W., and Ranges, H. A., Glomerular dynamics in the normal human kidney. J. Clin. Invest., 1940, 19, 751.

15. Smith, W. W., Finkelstein, N., and Smith, H. W., Renal excretion of hexitols (sorbitol, mannitol and dulcitol) and their derivatives (sorbiton, isomannide and sorbide) and of endogenous creatininelike chromogen in dog and man. J. Biol. Chem., 1940, 135, 231. 